Case Report

\title{
RESISTANT CANDIDA PNEUM ONIA IN TWO PATIENTS WITH CHRONIC KIDNEY DISEASE
}

\author{
Baikunje $S^{1}$, Upadhyaya $\mathrm{VS}^{2}$, Hosmane $\mathrm{GB}^{3} \&$ Rama Prakasha $\mathrm{S}^{4}$ \\ ${ }^{1}$ Associate Professor \& HOD, Department of Nephrology, ${ }^{2,3,4}$ Assistant Professors, ${ }^{2}$ Department of Radiology, \\ ${ }^{3}$ Pulmonary Medicine, ${ }^{4}$ Department of Medicine, K.S. Hegde M edical Academy, \\ Nitte University, M angalore - 575 018, India. \\ Correspondence : \\ Shashidhar Baikunje \\ Associate Professor \& HOD, Department of Nephrology, K.S. Hegde Medical Academy, \\ Nitte University, M angalore - 575 018, India. \\ E-mail: baikunje@hotmail.com
}

\begin{abstract}
:
Patients with chronic kidney disease are at increased risk of sepsis and infection is the second leading cause of death in dialysis patients. Primary candida pneumonia is extremely rare and there is very little data on its treatment and outcome in chronic kidney disease patients. We report resistant candida pneumonia in two patients with chronic kidney disease. Both patients were successfully treated with anidulafungin which is a newer echinocandin. The current recommendation is not to treat candida isolated from sputum or bronchoalveolar lavage specimens. But our cases demonstrate that in the relevant clinical setting, positive sputum cultures cannot be ignored and prompt treatment can be lifesaving in this condition with high mortality. Both the patients developed generalized pigmentation which resolved in a few weeks which, to the best of our knowledge has never been reported with this drug before. It is unknown whether this adverse effect is limited to patients with chronic kidney disease.
\end{abstract}

Keywords: Anidulafungin, Candida pneumonia, Chronic Kidney Disease.

\section{Introduction:}

Patients with chronic kidney disease (CKD) are at higher risk of infection compared to general population. Infectionrelated hospitalizations contribute substantially to excess morbidity and mortality in patients on dialysis, and infection is the second leading cause of death in this population. ${ }^{(1)}$ Patients who have CKD and do not require renal replacement therapy also seem to be at higher risk of infection compared with patients without CKD; however, data about such patients are very limited. Annual mortality rates in the dialysis population are increased by 10 -fold for pneumonia and 100-fold for sepsis compared with the general population ${ }^{(2)}$. There is sparse data on primary

Access this article online Quick Response Code

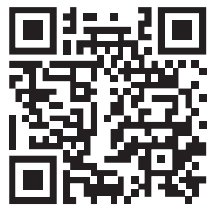
candida pneumonia in CKD patients and their outcome.

Case 1: A 54 year old male with adult polycystic kidney disease was admitted with fever, breathlessness and productive cough of four days duration. He had clinical and radiological evidence of left lower lobe pneumonia. (Fig.1a). His baseline creatinine was $6 \mathrm{mg} / \mathrm{dl}$ (M DRD eGFR $10 \mathrm{ml} / \mathrm{min} / 1.73 \mathrm{~m}^{2}$ ) which had deteriorated to $11.96 \mathrm{mg} / \mathrm{dl}$ (eGFR $\left.4 \mathrm{ml} / \mathrm{min} / 1.73 \mathrm{~m}^{2}\right)$. His haemoglobin was $7.2 \mathrm{~g} / \mathrm{dl}$ and total leucocyte count was 13,900 /cu.mm with neutrophil count of $90 \%$. He was initiated on haemodialysis. He was empirically treated with azithromycin and cefoperazone/sulbactum combination and the latter was changed to meropenem due to lack of response. After six days of antibiotic therapy he continued to be pyrexial, hypoxic and his $x$-ray showed no change. He grew heavy growth of candida (species not specified) in his sputum which was resistant to triazole group of antifungals. Blood culture was negative. He was initiated on anidulafungin which lead to complete radiological resolution in two weeks (Fig.1b). He tolerated the treatment very well but developed generalized hyperpigmentation which resolved after a month of discontinuing the drug. He remained on long term maintenance dialysis. 
Case 2: A 74 year old male with long standing hypertension and CKD stage 3 (baseline creatinine $1.8 \mathrm{mg} / \mathrm{dl}$, MDRD eGFR $37 \mathrm{ml} / \mathrm{min} / 1.73 \mathrm{~m}^{2}$ ) presumably secondary to hypertensive nephrosclerosis was admitted with right upper quadrant pain and fever since three days. His serum creatinine had deteriorated to 3.13(MDRD eGFR 20 $\mathrm{ml} / \mathrm{min} / 1.73 \mathrm{~m}^{2}$ ). His haemoglobin was $12.9 \mathrm{~g} / \mathrm{dl}$ and he had neutrophilic leucocytosis with total count of 19600 / cu.mm with $92 \%$ neutrophils. Liver function tests were mildly deranged with total bilirubin of $2.2 \mathrm{mg} / \mathrm{dl}$ (Direct 1 $\mathrm{mg} / \mathrm{dl}$, Indirect $1.2 \mathrm{mg} / \mathrm{dl}$ ), ALT of $54 \mathrm{U} / \mathrm{l}$ with normal AST and alkaline phosphatase. Ultrasound scan showed distended gall bladder with multiple calculi with minimal pericholecystic collection. A diagnosis of acute calculous cholecystitis was made. He had mild hypoxia and preoperative chest radiograph showed atelectasis at the right base. This was thought to be secondary to his abdominal pathology. He was empirically treated with piperecillin/tazobactum and metronidazole and he underwent open cholecystectomy on day three of admission under general anaesthesia. Postoperatively he had pyrexia, neutrophilic leucocytosis and hypoxia. He developed bibasal consolidation with moderate pleural effusion on the right which was aspirated to exclude empyema. His kidney function deteriorated and he was started on haemodialysis. Sputum culture grew heavy growth of resistant candida (species not specified). His blood culture was sterile but pus from the gall bladder grew pseudomonas which was fully sensitive. In spite of the appropriate antibiotic cover for six days he had persistant pyrexia, neutrophilic leucocytosis, worsening hypoxia and chest $x$-ray changes. He was initiated on anidulafungin with excellent clinical response. His fever, neutrophilia and hypoxia improved, kidney function recovered back to baseline but complete radiological resolution took four weeks. This patient also developed generalized hyperpigmentatation which completely resolved in about four weeks after he finished the antifungal therapy.

\section{Discussion :}

Pneumonia due to candida is extremely rare. There is very little literature available on candida pneumona in CKD

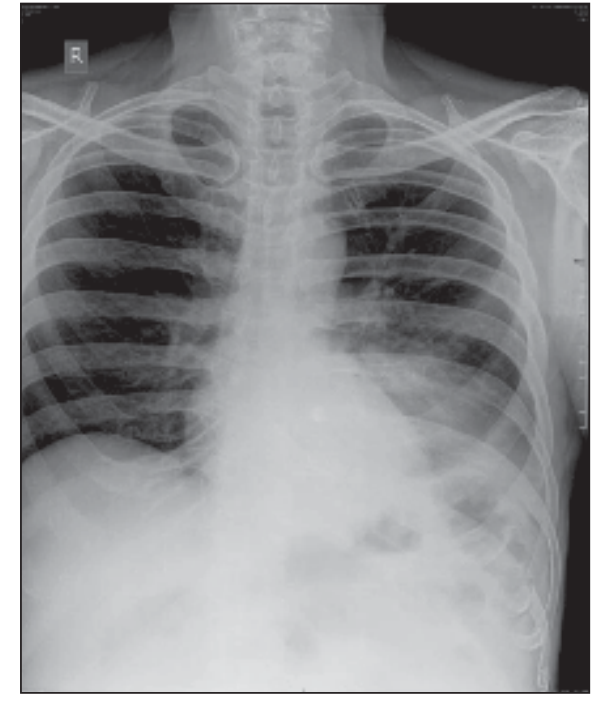

Figure 1a : Chest radiograph on admission shows left lower lobe consolidation.

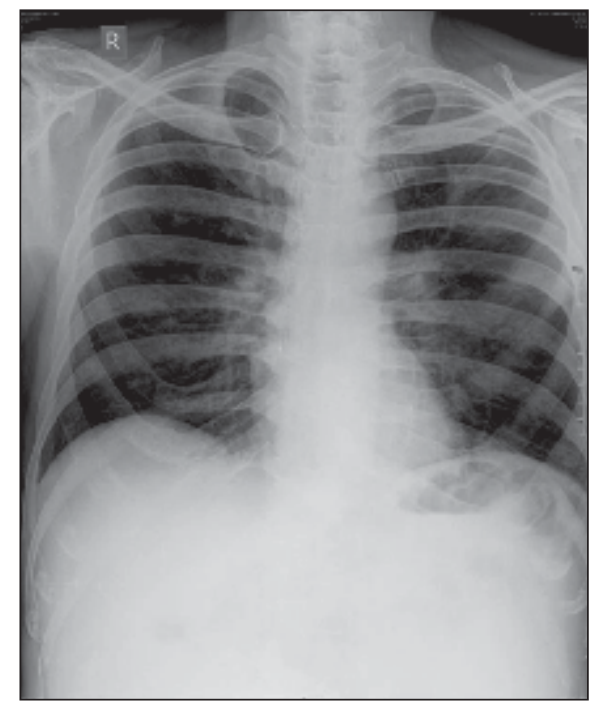

Figure 1b : Follow up radiograph in two weeks which shows complete resolution.

patients. The only accepted criterion for the definitive diagnosis of candida pneumonia is histologic demonstration of the fungus in lung tissue because positive cultures cannot distinguish between true infection and either colonization or sample contamination with oropharyngeal secretions. ${ }^{(3)} \mathrm{A}$ retrospective analysis from Spain concluded that nonneutropenic patients with isolation of candida species from bronchoscopic samples, even in high concentrations, are unlikely to have invasive candidiasis. ${ }^{(4)}$ Another study showed that for sputum culture, the sensitivity, specificity, and the positive and negative predictive values were $85 \%, 60 \%, 42 \%$, and $93 \%$, 
respectively; for bronchoalveolar lavage culture, these values were $71 \%, 57 \%, 29 \%$, and $89 \%$, respectively ${ }^{(5)}$. However, sputum culture was the key in initiating therapy in both our patients who had negative bacteriological cultures and no response to broad spectrum antibiotics.

The most common thin-section CT findings of pulmonary candidiasis are multiple nodular opacities, often bilateral which was present in $88 \%$ of patients in a study which included 17 hematopoietic stem cell transplant recipients with proven pulmonary candidiasis. ${ }^{(6)}$ Areas of air-space consolidation were identified in $65 \%$ patients and areas of ground-glass opacity were seen in 35\%. Even though CT scan was not carried out, one of our patients showed changes of unilateral lower lobe consolidation and the other patient had bilateral lower lobe consolidation with right sided effusion on chest $x$-ray. Pleural effusion was reported in 3 out of 17 patients in the above mentioned study.

Very little data is available on the therapy and outcome of patients with candida pneumonia and none in the CKD population. The current recommendation is not to treat candida isolated from sputum or BAL specimens. ${ }^{(7)}$ But our cases demonstrate that in the relevant clinical setting,

\section{References:}

1. US Renal Data System: USRDS 2007 Annual Data Report: Atlas of Chronic Kidney Disease and End-Stage Renal Disease in the United States, Bethesda, National Institutes of Health, National Institute of Diabetes and Digestive and Kidney Diseases, 2007.

2. Sarnak MJ, Jaber BL: Pulmonary infectious mortality among patients with end-stage renal disease. Chest 2001; 120:1883.

3. Haron E, Vartivarian S, Anaissie E, Dekmezian R, Bodie GP. Primary Candida pneumonia. Experience at a large cancer center and review of the literature. Medicine (Baltimore) 1993;72:137.

4. Rello J, Esandi ME, Díaz E, M ariscal D, Gallego M, Valles J. The role of Candida sp isolated from bronchoscopic samples in nonneutropenic patients. Chest 1998;114:146.

5. Kontoyiannis DP, Reddy BT, Torres HA, Luna M, Lewis RE, Tarrand J, Bodey GP, Raad II. Pulmonary candidiasis in patients with cancer: an autopsy study. Clin Infect Dis 2002;34:400.

6. Franquet T, Müller NL, Lee KS, Oikonomou A, Flint JD. Pulmonary candidiasis after hematopoietic stem cell transplantation: thin-section CT findings. Radiology. 2005;236:332.

7. Pappas PG, Kauffman CA, Andes D, Benjamin DK Jr, Calandra TF, Edwards JE Jr, Filler SG, Fisher JF, Kullberg BJ, Ostrosky-Zeichner L, Reboli AC, RexJH, Walsh TJ, Sobel JD. Clinical practice guidelines for the management of candidiasis: 2009 update by the Infectious Diseases Society of America. Clin Infect Dis 2009;48:503.

8. Reboli AC, Rotstein C, Pappas PG, Chapman SW, Kett DH, Kumar D, Betts R, Wible M, Goldstein BP, Schranz J, Krause DS, Walsh TJ. Anidulafungin Study Group:Anidulafungin versus fluconazole for invasive candidiasis. N Engl J M ed. 2007;356:2472. positive sputum cultures cannot be ignored and prompt treatment can be lifesaving in this condition with high mortality which can be as high as $84 \%{ }^{(3)}$. Since 2004 , several new antifungal agents have become available which are effective against candida resistant to azoles. Anidulafungin, a newer echinocandin, has potent activity against candida species and it showed superior clinical and microbiologic response compared with fluconazole. ${ }^{(8)}$ But the experience with this drug in the setting of CKD is limited and in our cases it was well tolerated except the reversible generalized skin pigmentation which both the patients developed. To our knowledge, this has never been reported before.

To conclude, fungal pneumonia needs to be considered in any immunosuppressed individual presenting with pneumonia poorly responding to standard treatment. Sputum culture is considered not very helpful in the diagnosis of candida pneumonia but in the relevant clinical setting, positive culture should not be ignored. Finally, even though anidulafungin can be extremely effective and well tolerated, reversible skin pigmentation can be an adverse effect. Whether this side effect is limited to patients with CKD is unknown and more studies are needed to address this issue. 